\title{
Combining immunotherapy and radiotherapy in lung cancer
}

\author{
Neeraj Bhalla ${ }^{1}$, Rachel Brooker ${ }^{1}$, Michael Brada ${ }^{1,2}$ \\ ${ }^{1}$ Clatterbridge Cancer Centre NHS Foundation Trust, Bebington, Wirral, Merseyside, UK; ${ }^{2}$ Department of Molecular and Clinical Cancer \\ Medicine, University of Liverpool, Liverpool, UK \\ Contributions: (I) Conception and design: All authors; (II) Administrative support: All authors; (III) Provision of study materials or patients: All \\ authors; (IV) Collection and assembly of data: All authors; (V) Data analysis and interpretation: All authors; (VI) Manuscript writing: All authors; (VII) \\ Final approval of manuscript: All authors. \\ Correspondence to: Dr. Neeraj Bhalla. Clatterbridge Cancer Centre NHS Foundation Trust, Bebington, Wirral CH63 4JY, UK.
}

Email: neerajbhalla@nhs.net.

\begin{abstract}
Immunotherapy has become standard of care in advanced non-small cell lung cancer (NSCLC) in a number of settings. Radiotherapy remains an important and potentially curative treatment for localized and locally advanced NSCLC not amenable to surgery. While the principal cytotoxic effect of ionizing radiation is via DNA damage, the effect on tumour microenvironment, promoting dendritic cell presentation of tumour-derived antigens to $\mathrm{T}$ cells stimulating the host adaptive immune system to mount an immune response against tumours cells, has become of particular interest when combining immunomodulating agents with radiation. The 'abscopal effect' of radiation where non-irradiated metastatic lesions may respond to radiation may be immune-mediated, via radiation primed anti-tumour $\mathrm{T}$ cells. Immune priming by radiation offers the potential for increasing the efficacy of immunotherapy and this is subject to on-going clinical trials underpinned by immunological bioassays. Increasing understanding of the interaction between tumour, radiation and immune cells at a molecular level provides a further opportunity for intervention to enhance the potential synergy between radiation and immunotherapy. Applying the potential efficacy of combination therapy to clinical practice requires caution particularly to ensure the safety of the two treatment modalities in early phase clinical trials, many of which are currently underway. We review the biological basis for combining radiation and immunotherapy and examine the existing pre-clinical and clinical evidence and the challenges posed by the new combination of treatments.
\end{abstract}

Keywords: Ionizing radiation; immunotherapy; non-small cell lung cancer (NSCLC)

Submitted May 10, 2018. Accepted for publication May 11, 2018.

doi: $10.21037 /$ jtd.2018.05.107

View this article at: http://dx.doi.org/10.21037/jtd.2018.05.107

\section{Introduction}

Lung cancer remains a major challenge as the most common cause of cancer death and the cause of significant morbidity with a clear need to improve outcomes. Radiotherapy remains an important and potentially curative treatment for localized and locally advanced non-small cell lung cancer (NSCLC) not amenable to surgery. While traditionally radiotherapy has been combined with chemotherapy to improve outcome, in the changing world of increasing use of immunotherapy as an alternative and a more effective systemic treatment, it is important to exploit the potential for combining the efficacy of radiation with immunotherapy. We explore the biological basis for potential interaction between radiotherapy and immunotherapy and review the current clinical evidence to assess the potential value of combining the two treatment modalities.

\section{Cancer immunoediting}

The concept of 'cancer immunosurveillance' (1), first proposed over a century ago (2), was investigated in the mid- $20^{\text {th }}$ century without apparent success. However, advances in immunology in the 1990s demonstrated in 
mouse models that endogenous components of the host immune system [interferon- $\gamma(\operatorname{IFN}-\gamma)](3,4)$ and lymphocytes could play a role in a cancer immunosurveillance process (5).

Current evidence supports the notion that each stage in the development and progression of cancer is the result of 'cross-talk' between the tumour and the host's immune system (6), described as the three 'Es' of immune-editingelimination, equilibrium and escape phases (7).

In the elimination phase incipient tumour is detected by the innate immune system where natural killer cells and $\gamma \delta$-T cells produce IFN- $\gamma$ that promotes cytotoxic activity of macrophages leading to destruction of developing cancer cells (8). Tumour cell death releases tumour-associated antigens that are taken up by dendritic cells, bone marrow derived professional antigen-presenting cells, which then undergo a process of maturation. The dendritic cells crosspresent the tumour peptide antigens to $T$ cells, stimulating activation of the adaptive immune system and causing proliferation of cytotoxic $\mathrm{T}$ cells, specific to the tumour antigen, leading to tumour cell death. The immune system also produces pro-inflammatory cytokines and tumour cell death produces 'danger signals'; these further activate tumour-specific $\mathrm{T}$ cells to cause tumour cell death (9).

In the equilibrium phase incomplete elimination and immune selection leave surviving tumour cells that may generate escape 'mutants' which lack tumour specific antigens, resulting in a balance between proliferation and cell death (sub-clinical tumour) (8).

Escape phase sees the emergence of tumour cells that are resistant to immune rejection by the host's innate immune system. An immunosuppressive microenvironment is created with tumour cells releasing inhibitory cytokines, IL-10 and TGF-B (10). Regulatory T cells (Tregs, formerly suppressor $\mathrm{T}$ cells) are recruited, tumour-related antigens are downregulated and MHC I expression suppressed (11), which ultimately leads to evasion.

Understanding of the molecular biology underpinning the interplay between tumour cells and the innate immune system leads naturally to investigation of therapeutic options, exploiting the knowledge of these molecular pathways.

\section{Immune checkpoints and their blockade by immunotherapeutic agents}

$\mathrm{T}$ cell activation and response is regulated by immune checkpoints-a system of checks and balances that, under physiological conditions, prevent autoimmune disease, but also protect tissues from excessive damage when the immune system is mounting an appropriate response against a foreign pathogen $(12,13)$. The expression of immune checkpoint proteins can be dysregulated by tumours and is part of the mechanism of 'escape' in cancer immune editing as well as a mechanism of resistance once tumour is established. The two immune-checkpoint receptors that have been most studied in cancer immunotherapy are cytotoxic T-lymphocyte-associated antigen 4 (CTLA-4) and programmed cell death protein 1 (PD-1).

CTLA-4 is an inhibitory $\mathrm{T}$ cell receptor limiting activation. It counterbalances a stimulatory $\mathrm{T}$ cell receptor, CD28 that is activated by binding of B7 ligands, residing on antigen presenting cells. Upon MHC presentation of an antigen, co-stimulation of CD28 results in T cell activation. CTLA-4 also binds and is activated by the same B7 ligands (with greater affinity than CD28) but, as it is an inhibitory receptor, ligand binding has the opposite effect, dampening the $\mathrm{T}$ cell response. Within the immunosuppressive environment of cancer this mechanism is exploited and CTLA-4 is over stimulated by the constant presentation of tumour-associated antigens leading to $\mathrm{T}$ cell exhaustion. CTLA-4 is also expressed by tumour recruited T-regs which again leads to $\mathrm{T}$ cell suppression and production of IL-10. Ipilimumab, a monoclonal antibody against CTLA-4, blocks this inhibitory pathway and restores effective $\mathrm{T}$ cell function. It was first approved for use by the FDA in 2011 following the publication of the landmark trial by Hodi et al. (14) in patients with previously treated metastatic melanoma, showing an overall survival (OS) advantage when compared to glycoprotein 100 vaccine. Its efficacy in lung cancer is currently limited to a phase II study where Ipilimumab is combined with paclitaxel and carboplatin chemotherapy as first line treatment in NSCLC. The study showed improved progression-free survival (PFS) with phased dosing (15); phase III trials are underway.

PD-1 is an inhibitory $T$ cell receptor that is induced once $\mathrm{T}$ cells are activated. Its main function is to act as a 'stop signal' (13), limiting immune responses in peripheral tissues to protect them from damage (16). PD-1 can be induced on other activated non-T lymphocyte subsets including $\mathrm{B}$ cells and natural killer cells (17). PD-L1 and PD-L2 are the stimulatory ligands for the inhibitory PD-1 receptor. PD-L1 is upregulated in solid tumours and increasing degrees of expression are associated with a poorer prognosis (18). By blocking the PD-1/PD-L1 axis the immunosuppressive environment is reversed and an antitumour immune response prevails. 
Pembrolizumab and nivolumab, monoclonal antibodies that block PD-1, have been shown in phase III trials to prolong time to progression and survival compared to palliative chemotherapy, in both treatment-naïve patients with advanced NSCLC $(19,20)$ and as second line treatment following progression after chemotherapy $(21,22)$.

Despite the optimism these immunotherapeutic agents have brought to the treatment of NSCLC, response rates remain between $19 \%$ and $50 \%$ (19-22) and lung cancer survival remains poor. Evidence is emerging of two distinct tumour phenotypes (23) that may partly explain non-response to immune-checkpoint blockers. A subset of patients with advanced solid tumours that show evidence of a $\mathrm{T}$ cellinflamed tumour microenvironment, have a more favourable response to immunotherapy $(24,25)$ and the presence of tumour-infiltrating lymphocytes (TIL) (26) is associated with better prognosis (24). Tumours with a high proportion of TILs are often referred to as 'hot' tumours (26). The ratio between numbers of cytotoxic $\mathrm{CD}^{+} \mathrm{T}$ cells and T-regs (suppressor $\mathrm{T}$ cells) in tumours also effects which tumours are responsive to immunotherapy (26).

'Cold' tumours describe a phenotype of solid tumours with scanty $\mathrm{T}$ lymphocytes, in which immune-checkpoint blockade with drugs such as ipilimumab and pembrolizumab is ineffective $(25,27)$. Correlation is seen between the low pre-treatment levels of $\mathrm{CD} 8^{+} \mathrm{T}$ cells in the tumour microenvironment and poor response to treatment (27). Careful density measurements of the different classes of infiltrating cytotoxic T cells and helper cells (Th1, Th2) in the pre-existing immune microenvironment of colorectal cancers have allowed quantification of the different immune cell types present (28). This is described as the 'Immunoscore' (29) and may be a prognostic predictor of outcome following treatment (29). Interventions that will allow immunotherapeutic agents to become effective in 'cold' tumours are likely to be key in improving the modest response rates to these agents.

\section{Mechanism of radiation-induced cell death}

DNA is the principal target for cell damage by ionizing radiation, leading to cell death (30). Radiation induced DNA lesions in the form of base damage, single-strand breaks or double-strand breaks (31) occur through direct ionization or indirectly by the formation of hydroxyl free radicals.

Normal cell DNA repair mechanisms tend to maintain the fidelity of the DNA in normal functioning of the cell. Defective DNA damage response and repair mechanisms in cancer cells may lead to cell death that is triggered following radiation induced cell stress (32). Radiation induced bystander effect may also contribute $(33,34)$.

\section{Immunological consequences of radiation- induced cell death}

In addition to killing tumour cells radiation stimulates changes in the tumour microenvironment. It can have immunosuppressive effects (35). Localised radiotherapy recruits immunosuppressive myeloid cells that directly promote tumour growth (36) and contributes to an immunosuppressive environment by making $\mathrm{T}$ cells dysfunctional (37). Radiotherapy also up-regulates PD-L1 expression in the tumour microenvironment that activates the inhibitory PD-1 receptor on $\mathrm{T}$ cells, inhibiting a $\mathrm{T}$ cell anti-tumour response (38).

However, radiation may also have positive immunemodulatory effects leading to the generation of a beneficial tumour-specific immune response (39). During tumour cell death due to ionizing radiation, tumour antigens are released and presented to dendritic cells that activate the adaptive immune system, stimulating proliferation of $\mathrm{T}$ cells which then mount a tumour-specific response $(40,41)$. This anti-tumour response leads to radiation-induced 'immunogenic cell death' (42).

The molecular mechanisms by which dendritic cells are activated by ionizing radiation are being unravelled. Upregulation and translocation of calreticulin within dying, irradiated tumour cells, which is then expressed on the cell membrane, allows dendritic cells to recognize the dying cells and engulf them by phagocytosis (recognition signal) $(39,43,44)$. Also, release of high-mobility group protein B1 (non-histone nuclear protein) from dying, irradiated tumour cells binds to Toll receptor 4, leading to dendritic cell activation ('danger' signal) $(44,45)$.

Activated dendritic cells migrate to local lymph nodes where naïve $T$ cells are presented with antigens specific to the tumour and are stimulated. These effector $\mathrm{T}$ cells migrate back to the tumour, attracted by chemokines induced by tumour irradiation $(46,47)$. Surviving irradiated cells display enhanced expression of intercellular adhesion molecule (ICAM-1), death receptor Fas and major histocompatibility complex class I antigen-presenting molecules which all improve their recognition and killing by the activated anti-tumour T cells (48). This mechanism by which the irradiated tumour becomes a source of tumour antigens is described as in-situ auto-vaccination $(8,49,50)$ 
and this appears to have a role in both the priming and effector phases of anti-tumour immunity (44). In this way, radiation can prime the innate immune system and promote an anti-tumour response, recruiting $\mathrm{T}$ lymphocytes and turning 'cold' tumours 'hot', meaning they are more likely to respond to systemic immunotherapy.

Radiotherapy-induced immunogenic cell death therefore relies on T lymphocytes, which is at odds to the fact that the radiosensitivity of $T$ lymphocytes makes them vulnerable targets during radiation. Radiation can induce lymphopenia by killing not only TIL but also lymphocytes in peripheral blood that transit through the radiation field. Severe lymphopenia has been shown to be associated with a poor prognosis in NSCLC (51). Limiting the size of radiation fields is therefore a consideration when investigating combination radiotherapy and immunotherapy as most immunotherapies depend on functioning $\mathrm{T}$ lymphocytes.

'Abscopal effect' where non-irradiated metastatic lesions outside the radiation field apparently respond to treatment may also be immune-mediated $(49,52)$. The theory is that radiation triggers anti-tumour $\mathrm{T}$ cell responses that can kill tumour cells distant to the irradiated tumour (49). Immune priming by ionizing radiation is insufficient to mount a strong and durable $\mathrm{T}$ cell response that is capable of eradicating the irradiated tumour and established metastases (44). The abscopal and immune-modulatory effects of ionizing radiation can be exploited by combining radiotherapy with systemic immunotherapy and this is currently explored in clinical studies.

\section{Pre-clinical evidence}

Interplay between radiation therapy and immunotherapy has been demonstrated in preclinical studies (53). The National Cancer Institute (NCI) Translational Research Working Group defined immune response modifiers (IRM) as 'immunotherapy agents that mimic, augment, or require participation of the host immune system for optimal effectiveness' (54). A variety of IRM have been used in experimental models targeting different aspects of the immune pathway.

Interleukin-2 (IL-2), a T-cell growth factor, may work synergistically with radiation to produce anti-tumour effects (55). Other cytokines, including IL-3, IL-12 and tumour necrosis factor, have been tested in combination with radiation with positive outcomes in mouse models $(56,57)$. However, due to significant toxicities interleukins have had limited clinical use in combination with radiation.

Building on the theory of in-situ auto-vaccination experiments have examined vaccination with autologous tumour cells, modified to be more immunogenic, in combination with radiotherapy. In mouse glioma models the combination resulted in induction of anti-tumour $\mathrm{T}$ cell response resulting in prolonged survival in a mouse model (58).

Flt3-L growth factor stimulates dendritic cell production and has a role in inducing antitumor immunity in mouse tumours. Irradiation of the primary tumour in a murine model to 60 Gy, followed by Flt3-L administration, prevented development of lung metastases and prolonged diseasefree survival compared to radiation or Flt3-L alone (59). The addition of radiotherapy to an IRM causing an effect in non-irradiated tumour, greater than that seen with Flt3-L alone, is an example of abscopal effect. However, the need for such a high single radiation dose to obtain systemic antitumor response has limited clinical relevance (60).

In a mouse model using $67 \mathrm{NR}$ mammary carcinoma Flt3-L together with lower doses of radiation demonstrated immune-mediated abscopal effect (49). Mammary carcinoma xenografts in both flanks were treated with Flt3-L daily for 10 days after local radiation therapy to only one flank to a single dose of 2 or 6 Gy. Radiotherapy alone led to growth delay of the irradiated tumour alone. Flt3-L had no effect on either tumour (control). Simultaneous administration of Flt3-L and irradiation led to growth delay in both the irradiated and non-irradiated tumour. No growth delay was observed in the non-irradiated tumour in nude mice ( $T$ cell deficient), suggesting that $T$ cells are required to mediate distant tumour effect induced by radiation. Combining two flank 67NR tumours and A20 lymphoma in the same mouse and irradiating one 67NR tumour with Flt3-L administration did not result in growth delay of A20 lymphoma, suggesting tumour antigen specificity is important in the immune-mediated response (49). Injection of dendritic cells around the tumour after irradiation also increased anti-tumour response compared to radiation alone $(61,62)$.

Demaria et al. (63) used a metastatic mouse mammary carcinoma 4T1 model to test the combination of radiation and CTLA-4 blockade. Mice with subcutaneously implanted mammary carcinoma cell line, which spontaneously develops systemic metastases, were randomly assigned to radiation to the primary tumour alone, 9H10 monoclonal antibody (Mab) against CTLA-4 alone, and the combination of radiation and $9 \mathrm{H} 10 \mathrm{Mab}$. Mice treated with radiation and $9 \mathrm{H} 10 \mathrm{Mab}$ had prolonged survival 
compared to other groups and this was associated with fewer lung metastases which suggests immune-mediated abscopal effect-local radiation having a primer and effector role in a systemic immune response, augmented by CTLA-4 blockade. Depletion of $\mathrm{CD}^{+}{ }^{+} \mathrm{T}$-cell was not associated with a reduction in the number of lung metastases, suggesting that $\mathrm{CD} 8^{+} \mathrm{T}$ cells play a crucial role in the anti-metastatic effect of the combination of CTLA-4 blockade and radiotherapy (63). Increasing the radiation dose was associated with improved local tumour control, distant tumour control and survival.

In addition to the beneficial effect of radiation modulating the tumour microenvironment and releasing tumour specific antigens for $\mathrm{T}$ cell recognition, radiation also has immunosuppressive effects. Irradiation of pancreatic tumours in mice results in a greater proportion of induced macrophages with immunosuppressive phenotypes and more T-regulatory cell phenotypes reducing anti-tumour immune response (37). Treatment with macrophage colony-stimulating factor 1 (M-CSF) blockade leads to some inhibition of the immunosuppressive effect of radiation (63). Radiation also leads to an increase in CCL2 chemokine production causing recruitment of $\mathrm{Ly}_{6} \mathrm{C}^{+} \mathrm{CCR} 2^{+}$monocytes supporting tumour proliferation and neovascularization after radiotherapy. Combining radiotherapy with an anti-CCL2 antibody disrupted monocyte recruitment with a decrease in tumour proliferation and vascularization and a delay in tumour growth.

Similarly the combination of NHS-IL2, an antibody specific for necrotic DNA fused to modified human IL-2, and cisplatin with radiotherapy in an in vivo study of murine lung carcinoma was more effective than controls (64).

The experiments suggest that combination of radiation with IRM in animal models using homogenous cell lines is more effective than either modality alone. However, this may not be truly representative of the effect of the combination on heterogeneous human solid tumours (65). Such in vivo experiments also do not provide sufficient information on the potential toxicity of the combination particularly as the dose fractionation regimens used rarely parallel radiotherapy regimens used in the clinic. Nevertheless, the pre-clinical evidence suggests potential synergy, which can be explored in clinical trials.

\section{Clinical trials}

\section{Concurrent radiation-immunotherapy trials}

In a phase Ib trial of NHS-IL2, an antibody specific for necrotic DNA fused to modified human IL-2, patients with metastatic NSCLC, who achieved disease control after first line platinum-based chemotherapy, received palliative radiotherapy to the primary lung tumour or a pulmonary metastasis (20 Gy in 5 daily fractions), and 3-weekly cycles of NHS-IL2 until significant toxicity or disease progression. The commonest reported adverse effects were fatigue, anorexia and rash occurring even in the lowest NHS-IL2 dose cohort and similar to toxicities observed with NHS-IL2 monotherapy. Grade 3 toxicities included anaemia, lymphopenia and dose-limiting pericarditis. While pneumonitis, as a potential toxicity of both radiotherapy and immunotherapy, was not reported, four patients had unexplained dyspnoea. The treatment was associated with a transient increase in leukocyte count and an increase of both proliferating and memory CD4 and CD8 T cells during the first cycle of treatment. No objective clinical responses were observed. The median PFS was 2.9 months and median OS was 8.6 months; there were two longer-term survivors. NHS-IL2 with radiation was therefore reasonably well tolerated with a suggestion of benefit in some patients (64).

In a phase II study, patients with chemo-refractory metastatic NSCLC received ipilimumab within 24 hours of starting palliative radiotherapy (30 Gy in 5 fractions) to one metastatic lesion, with ipilimumab repeated every 3 weeks for 4 cycles. In an early report when 12 patients were evaluable, complete response was seen in 2 patients, stable disease or partial response in 3 and progressive disease in 7 patients. Toxicities included rash (including G3), fatigue and diarrhoea. One patient experienced G2 pneumonitis following irradiation of a lung lesion. The combination treatment could be tolerated but toxicity was significant, even in such a small group of patients (66).

A phase II study (RTOG99-09) of postoperative adjuvant vaccine immunotherapy (m 11D10 anti-idiotype vaccine and 3H1 anti-idiotype vaccine) and concurrent radiation in patients with completely resected stage II and stage IIIA NSCLC, tested humoral and T-cell responses, in addition to toxicity and tumour control endpoints and the results are awaited.

\section{Sequential radiation-immunotherapy trials}

A phase I/II study examined the combination of recombinant MAGE-A3 protein, formulated with the immune-stimulant AS15, in synergy with radiotherapy in 1 of 4 of the study cohorts. MAGE-A3 is considered to be a tumour-specific antigen expressed on $24-45 \%$ 
of NSCLCs (67). Cohorts 1, 2 and 3 used MAGE-A3 as adjuvant therapy following resection of early stage NSCLC concurrent with cisplatin/vinorelbine chemotherapy, sequential to chemotherapy or as adjuvant monotherapy respectively. Patients with unresectable stage III tumours were entered into cohort 4 and received at least 2 cycles of chemotherapy followed by sequential radiotherapy and then MAGE-A3 immunotherapy, administered intramuscularly every 3 weeks for 8 doses. Of 12 patients in the radiotherapy group (cohort 4), 11 experienced one or more G1-2 adverse effects. These included skin reactions and flu-like symptoms in 8 patients, infections in 7 and respiratory side effects in 5 patients. One patient in cohort 4 died of bronchial haemorrhage. The incidence of adverse effects in patients treated with radiotherapy prior to MAGE-A3 was no different compared to MAGE-A3 alone.

Incomplete immunogenicity results show that prior to immunotherapy, $9 \%$ of patients were seropositive for MAGE-A3-specific antibodies and $10 \%$ and $5 \%$ had detectable MAGE-A3-specific $\mathrm{CD}^{+}{ }^{+}$and $\mathrm{CD}^{+}{ }^{+} \mathrm{T}$-cell responses respectively. After treatment, MAGE-A3-specific $\mathrm{CD}^{+}$T-cell responses were induced in $29 \%$ (10/34) of patients with resected tumours (cohorts 1-3) and in $83.3 \%$ (5/6) patients with unresectable tumours who had received radiotherapy (cohort 4). The corresponding induced $\mathrm{CD} 8^{+}$ T-cell responses to immunotherapy were $6 \%$ (2/34; cohorts $1-3)$ and $33 \%(2 / 6$; cohort 4$)(67)$. The results in this small cohort of patients suggest potentiation of immune cell response in combination with radiotherapy that is in keeping with the role of radiation priming the immune system. A subsequent phase III trial examined the role of adjuvant MAGE-A3 (with or without chemotherapy) in completely resected NSCLC without prolongation of disease-free survival compared to placebo (68) leading to discontinuation of any further development of MAGE-A3 in NSCLC. The role of radiotherapy in synergy with MAGE-A3 has not been examined further.

A phase II cancer vaccine and radiotherapy study used the liposomal vaccine, L-BLP25 (tecemotide), a synthetic lipopeptide designed to induce a $\mathrm{T}$-cell response to the mucin 1 glycoprotein which is overexpressed and abnormally glycosylated in NSCLC (69). Patients with stage IIIB or IV NSCLC, achieving at least stable disease following chemoradiation or palliative chemotherapy respectively, were randomised to either L-BLP25 and best supportive care (BSC) or BSC. A single low intravenous dose of cyclophosphamide was administered 3 days before the first vaccine immunotherapy to enhance its effects and L-BLP25 was administered subcutaneously weekly for 8 weeks and could continue as 6-weekly maintenance injections at investigators discretion. The L-BLP25 was well tolerated with $97 \%$ completing the primary phase of L-BLP2 5 treatment and $69 \%$ continuing on the maintenance vaccine phase (70). The 3-year survival was $31 \%$ in the L-BLP25 arm and $17 \%$ in BSC arm $(\mathrm{P}=0.035)$. Subgroup analysis suggested that the survival benefit was confined to patients with localised stage IIIB disease with a 3 -year survival of $49 \%$ in the vaccine compared to $27 \%$ in the BSC group $(\mathrm{P}=0.07)$ with respective median survivals of 31 and 13 months. The suggestion is therefore that radiotherapy may have primed the immune response (71).

Tecemotide vaccine combined with radiotherapy was tested in a phase III randomised-controlled trial in locally advanced NSCLC (START trial) in which patients with unresectable stage III NSCLC, who had at least stable disease following chemoradiation treatment, were randomised between tecemotide maintenance vaccine (L-BLP25) and placebo. There was no significant difference in median OS [26 months (95\% CI: 22.5-29.2) versus 23 months (95\% CI: 19.6-25.5) respectively (adjusted HR 0.88, 0.75-1.03; $\mathrm{P}=0.123)$ ]. Patients were stratified according to concurrent or sequential chemoradiation. Patients treated with concurrent chemoradiotherapy had an improved outcome with a median survival of 31 months (95\% CI: 25.6-36.8) in the tecemotide arm, compared to 21 months (95\% CI: 17.4-23.9) in the placebo arm (adjusted HR $0.78,0.64-0.95 ; \mathrm{P}=0.016$ ) (72). This led to a phase III trial (START2) randomising patients with unresectable stage III NSCLC treated with primary concurrent chemoradiation to maintenance tecemotide vaccine or placebo.

Tecemotide investigated in patients with unresectable stage III NSCLC after primary chemoradiotherapy in Japan showed no benefit in OS or any secondary endpoints in a randomised phase II study (73) and further development of tecemotide, including the START 2 trial, were terminated. A phase II trial examining L-BLP25 in the same clinical setting, but with the addition of bevacizumab, remains in progress.

A phase III randomised-controlled, double-blinded trial (PACIFIC Trial) (74) using a PD-L1 inhibitor, durvalumab, following radiation, randomised patients with unresectable stage III NSCLC, achieving at least stable disease following concurrent chemoradiation, to 2-weekly intravenous durvalumab consolidation or placebo for up to 12 months, with a 2:1 randomisation. No threshold 
PD-L1 biomarker level was prerequisite to treatment. Planned interim analysis showed a significant improvement in both median and 18-month PFS rate in the durvalumab arm compared to placebo [16.8 months (95\% CI: $13.0-18.1$ ) versus 5.6 months (95\% CI: $4.6-7.8$ ), and $44.2 \%$ (95\% CI: $37.7-50.5 \%$ ) versus $27 \%$ (95\% CI: 19.9-34.5\%) respectively]. Pneumonitis and pneumonia were the commonest reasons for discontinuation of durvalumab or placebo: $33.9 \%$ (G3/4 3.4\%) and $24.8 \%$ (G3/4 2.6\%) for pneumonitis and 13.1\% (G3/4 4.4\%) and $7.7 \%$ (G3/4 3.8\%) for pneumonia, respectively. Adverse events that required concomitant steroid, endocrine or immunosuppressive treatment were reported in $42.1 \%$ and $17.1 \%$ of patients respectively. There were 21 deaths in the durvalumab arm and 13 in the placebo arm, including 4 and 3 with pneumonitis respectively.

The significant difference between the two arms demonstrated at interim analysis could be due to the adjuvant effect of additional immunotherapy. It is also possible that the prior radiation primes the immune system, thus improving the response to subsequent immunotherapy, even in patients who may have had 'cold', immunotherapyresistant tumours initially. However, the control arm has poorer than expected outcomes with a median PFS of only 5.6 months for stage III NSCLC following concurrent chemoradiation which is likely exaggerating any differences between the two arms. The addition of durvalumab significantly increases toxicity with one-third of patients having to discontinue the immunotherapy due to pneumonitis (74).

\section{Radiation and immunotherapy clinical trials in progress}

With PD-1 inhibitors, such as pembrolizumab and nivolumab, now becoming standard of care in a variety of settings in NSCLC, there has been an explosion of trials investigating their use with radiotherapy. The trials, investigate a number of questions from sequencing of treatment modalities to dose-fractionation. Table 1 shows some of the currently active trials combining radiation and immune-checkpoint inhibitors. They are predominantly phase 1 and 2 trials with a focus on the potential toxicity of combining checkpoint inhibitors with radiotherapy. As both lung radiotherapy and immunotherapy can cause pneumonitis the incidence and severity of pneumonitis of combined treatments will be of particular relevance.

A randomised I-SABR trial tests the addition of nivolumab to stereotactic radiotherapy in early lung cancer and solitary pulmonary metastases looking both at toxicity and efficacy in terms of radiation priming the response to immunotherapy with the potential for enhancing immunotherapy efficacy.

In the PembroX phase 2 trial patients with NSCLC planned for surgical resection (stages I-IIIA) are randomised to pre-operative pembrolizumab alone or pembrolizumab and radiotherapy with a single stereotactic fraction of $12 \mathrm{~Gy}$ delivered to $50 \%$ of the primary tumour. The resected tumour is then assessed for sub-types of $\mathrm{T}$ lymphocyte infiltration in the two arms testing the hypotheses of 'cold' tumours (lacking TIL) and potential radiotherapy priming to increase the response to immunotherapy.

Several studies investigate combination CTLA-4 and PD-1 inhibitors together with radiotherapy. As the combination of two or more checkpoint inhibitors in the treatment of metastatic melanoma is associated with increased toxicity, the combination of agents in patients with lung cancer and frequent comorbidity, together with radiotherapy may prove challenging.

\section{Summary}

Increased understanding of immune mechanisms at molecular and cellular level has revealed interactions between tumours, radiation and the immune system. DNA remains the principal target for ionising radiation damage leading to cell death. Although the effects of radiation on tumour microenvironment are becoming more understood, it is likely that current state of knowledge is only a glimpse into the complexity of immune interactions with more pathways involved in the interplay yet to be discovered.

The relative success of checkpoint inhibitors in NSCLC combined with the concept of 'priming' the immune system with radiation to make 'cold' less responsive tumours more responsive to immunotherapy, has opened the door to studies combining radiation with immunotherapy. While the published pre-clinical model studies are encouraging, it is difficult to be certain of their relevance, as model systems are unlikely to be representative of the complexity of the true in vivo setting.

Initial interest in vaccine immunotherapy combined with radiation has seen no clinical application of significant benefit. Studies of immune modulators such as interleukins and recombinant antigens in combination with radiation have not produced robust data on clinical outcome to draw any conclusions.

Current interest in combining radiation with immune 


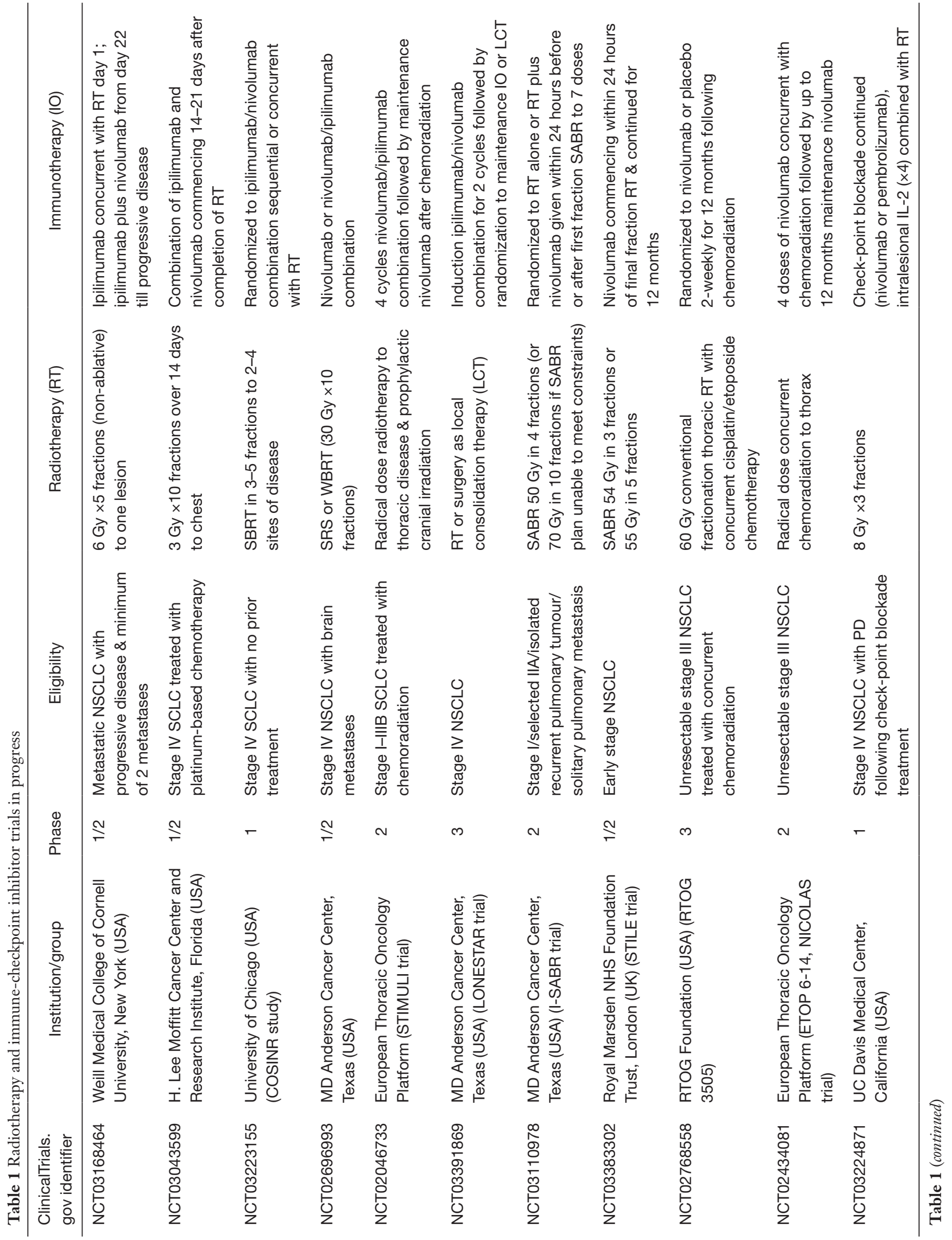




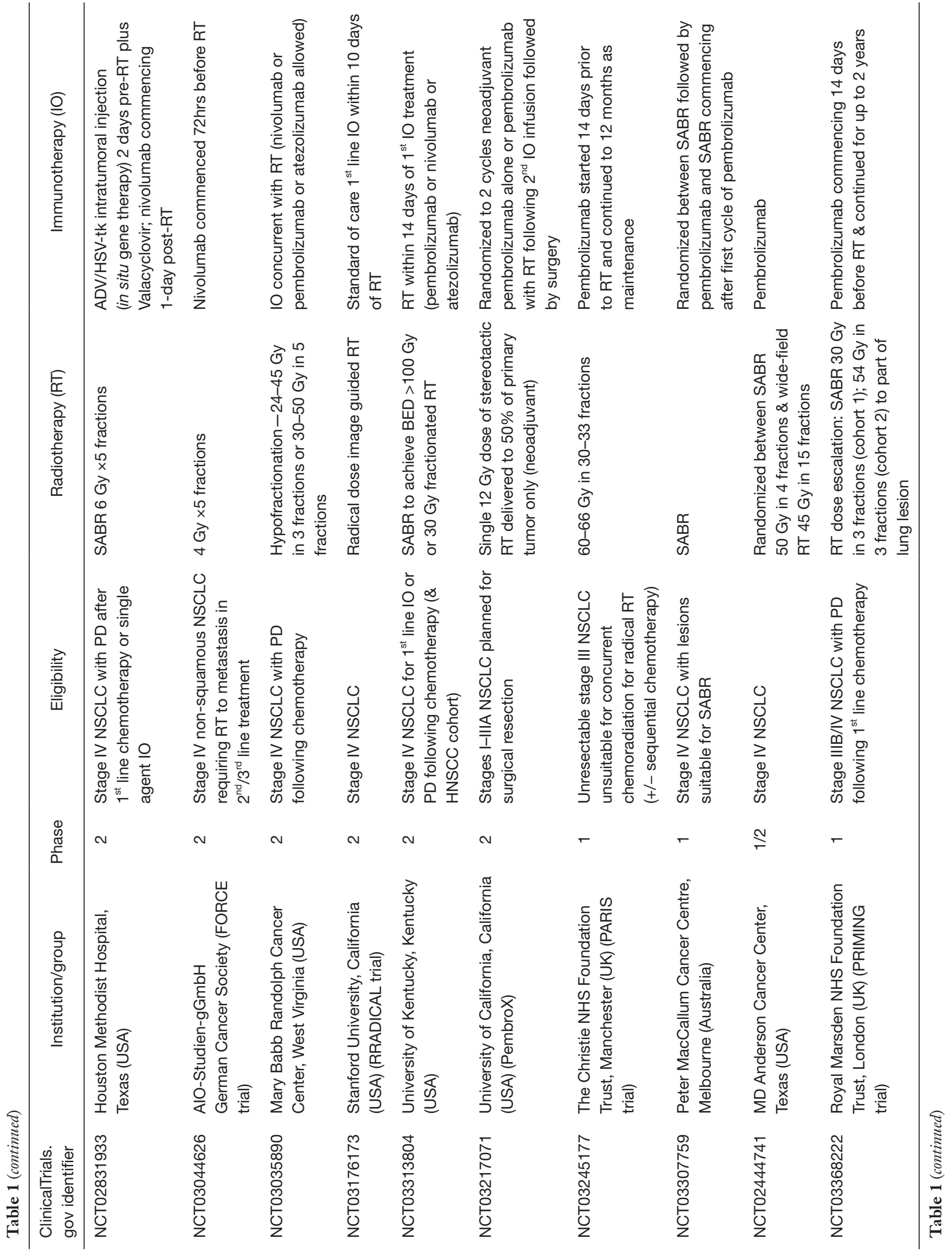




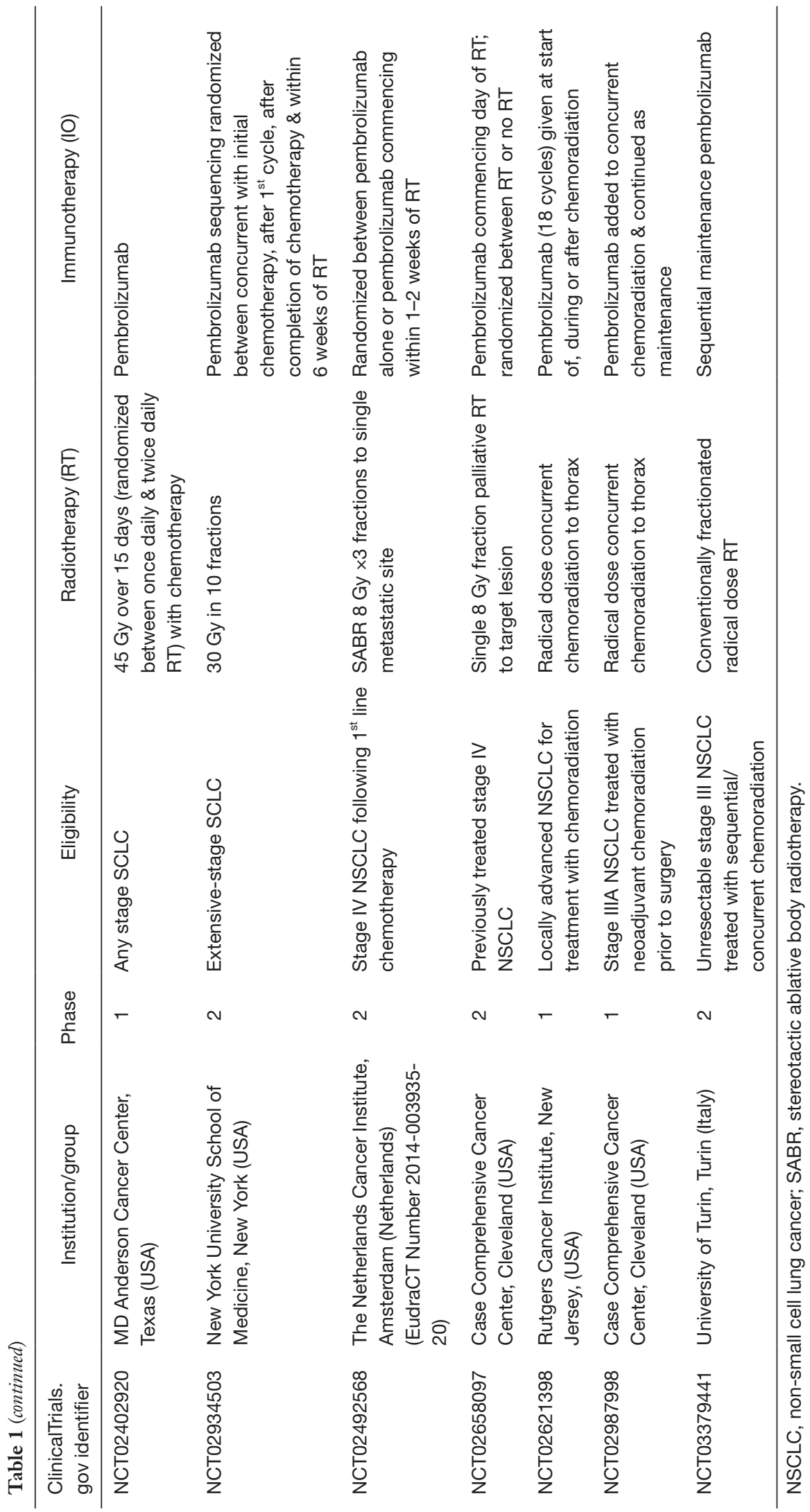


checkpoint inhibitors has a particular focus on NSCLC. Table 1 illustrates the range of questions requiring answers prior to embarking on large randomised trials of combined treatments. Optimum dose fractionation schemes required to prime the immune system are not defined and the studies largely reflect favoured radiation practice. At one end of the spectrum, immune checkpoint inhibitors have been combined with ablative dose of radiation (SABR) and at the other with low dose palliative regimens. The volume of irradiation to enhance the required immune modulation is also not defined; concerns of radiation causing lymphopenia and thereby reducing effectiveness of immunotherapies means partial target irradiation is utilised in some trials. Sequencing of radiation and immunotherapy is also not clear and trials examining this are under way.

Safety and toxicity are of paramount importance and combining data on adverse effects from all trials will be important to tease out rare but serious adverse effects and in the setting of NSCLC radiotherapy, to ascertain the potential risk of the combined effect of the two modalities on the incidence and severity of pneumonitis.

Biomarker studies, particularly focusing on measures of immune response and activity are an important component of current and future clinical trials to try and define molecular predictors of outcome. The complexity of immune interactions at cellular level means that many factors such as the timing of assays in relation to dosing and radiation and the type of cells of the immune system being assessed need to be considered and evaluated prior to employing tumour microenvironment and immune system assays as surrogates of treatment efficacy. As the immune functioning and response are dynamic it is likely that single snapshot assays will not be sufficient and there will be a need for dynamic testing which may also include the need for repeat biopsies unless surrogate serum or circulating cellular biomarkers are discovered. The potential exists for non-invasive monitoring of immune activity by dynamic imaging of labelled immune cells.

We are entering an exciting era of evolving cancer immunotherapy with a potential for radiation to enhance the effect of immune modulatory drugs. This is the beginning of a journey with a need for well-designed biomarker driven prospective studies of radiation and immunotherapy to answer an ever-increasing list of questions prior to embarking on the much-needed prospective trials testing the role of combination radiation-immunotherapy. The particular focus on lung cancer as one of the tumours with the most promising efficacy of immunotherapy poses novel challenges to a fast-developing landscape with a real hope of improving treatment outcomes.

\section{Acknowledgements}

This work was undertaken in the Clatterbridge Cancer Centre NHS Foundation Trust and the University of Liverpool and we are grateful for their support. The views expressed in this publication are those of the authors.

\section{Footnote}

Conflicts of Interest: The authors have no conflicts of interest to declare.

\section{References}

1. Burnet FM. The concept of immunological surveillance. Prog Exp Tumor Res 1970;13:1-27.

2. Ehrlich P. Ueber den jetzigen Stand der Karzinomforschung. Ned Tijdschr Geneeskd 1909;5:273-90.

3. Dighe AS, Richards E, Old L, et al. Enhanced in vivo growth and resistance to rejection of tumor cells expressing dominant negative IFN-receptors. Immunity 1994;1:447-56.

4. Kaplan DH, Shankaran V, Dighe AS, et al. Demonstration of an interferon gamma-dependent tumor surveillance system in immunocompetent mice. Proc Natl Acad Sci USA 1998;95:7556-61.

5. Smyth MJ, Thia KYT, Street SEA, et al. Differential tumor surveillance by natural killer (NK) and NKT cells. J Exp Med 2000;191:661-8.

6. Formenti SC, Demaria S. Combining radiotherapy and cancer immunotherapy: a paradigm shift. J Natl Cancer Inst 2013;105:256-65.

7. Dunn GP, Old LJ, Screiber RD. The three Es of cancer immunoediting. Annu Rev Immunol 2004;22:329-60.

8. Formenti SC, Demaria S. Radiation therapy to convert the tumor into an in situ vaccine. Int $\mathrm{J}$ Radiat Oncol Biol Phys 2012;84:879-80.

9. Schreiber RD, Old LJ, Smyth MJ. Cancer immunoediting: integrating immunity's roles in cancer suppression and promotion. Science 2011;331:1565-70.

10. Dong H, Chen L. B7-H1 pathway and its role in the evasion of tumor immunity. J Mol Med 2003;81:281-7.

11. DuPage M, Mazumdar C, Schmidt LM, et al. Expression of tumour-specific antigens underlies cancer 
immunoediting. Nature 2012;482:405-9.

12. Buchbinder EI, Desai A. CTLA-4 and PD-1 Pathways - Similarities, Differences and Implications of their Inhibition. Am J Clin Oncol 2016;39:98-106.

13. Pardoll DM. The blockade of immune checkpoints in cancer immunotherapy. Nat Rev Cancer 2012;12:252-64.

14. Hodi FS, O’Day SJ, Mc Dermott DF, et al. Improved survival with ipilimumab in patients with metastatic melanoma. N Engl J Med 2010;363:711-23.

15. Lynch TJ, Bondarenko I, Luft A, et al. Ipilimumab in combination with paclitaxel and carboplatin as first-line treatment in stage IIIB/IV non-small-cell lung cancer: results from a randomized, double-blind, multicenter Phase II study. J Clin Oncol 2012;30:2046-54.

16. Freeman GJ, Long AJ, Iwai Y, et al. Engagement of the PD-1 immunoinhibitory receptor by a novel B7 family member leads to negative regulation of lymphocyte activation. J. Exp. Med 2000;192:1027-34.

17. Benson DM, Bakan CE, Mishra A, et al. The PD-1/ PD-L1 axis modulates the natural killer cell versus multiple myeloma effect: a therapeutic target for CT011, a novel monoclonal anti-PD-1 antibody. Blood 2010 116:2286-2294.

18. Patel SP, Kurzrock R. PD-L1 expression as a predictive biomarker in cancer immunotherapy. Mol Cancer Ther 2015;14:847-56.

19. Reck M, Rodriguez-Abreu D, Robinson AG, et al. Pembrolizumab versus Chemotherapy for PD-L1Postive Non-Small-Cell Lung Cancer. N Engl J Med 2016;375:1823-33.

20. Carbone DP, Reck M, Paz-Ares L, et al. First-Line Nivolumab in Stage IV or Recurrent Non-Small-Cell Lung Cancer. N Engl J Med 2017;376:2415-26.

21. Herbst RS, Baas P, Kim D, et al. Pembrolizumab versus docetaxel for previously treated, PD-L1-positive, advanced non-small-cell lung cancer (KEYNOTE-010): a randomised controlled trial. Lancet 2016;387:1540-50.

22. Borghaei H, Paz-Ares L, Hom L, et al. Nivolumab versus Docetaxel in Advanced Nonsquamous Non-Small-Cell Lung Cancer. N Engl J Med 2015;373:1627-39.

23. Gajewski TF, Schreiber H, Fu YX. Innate and adaptive immune cells in the tumor microenvironment. Nat Immunol 2013;14:1014-22.

24. Gajewski TF. The next hurdle in cancer immunotherapy: overcoming the non-T cell inflamed tumor microenvironment. Semin Oncol 2015;42:663-71.

25. Ji RR, Cahsalow SD, Wand L, et al. An immuneactive tumor microenvironment favors clinical response to ipilimumab. Cancer Immunol. Immunother 2012;61:1019-31.

26. Demaria S, Coleman CN, Formenti SC. Radiotherapy: changing the game in immunotherapy. Trends Cancer 2016;2;286-94.

27. Tumeh PC, Harview CL, Yearley JH, et al. PD-1 blockade induces responses by inhibiting adaptive immune resistance. Nature 2014;515:568-71.

28. Mlecnik B, Bindea G, Angell HK, et al. Integrative analyses of colorectal cancer show immunoscore is a strong predictor of patient survival than microsatellite instability. Immunity 2016;44:698-711.

29. Galon J, Pages F, Marincola FM, et al. Cancer classification using the Immunoscore: a worldwide task force. J Transl Med 2012;10:205.

30. Ward JF. The complexity of DNA damage: relevance to biological consequences. Int J Radiat Biol 1994;66:427-32.

31. Ward JF. DNA damage produced by ionizing radiation in mammalian cells: identities, mechanisms of formation and reparability. Prog Nucleic Acid Res Mol Biol 1988;35:95-125.

32. Eriksson D, Stigbrand T. Radiation-induced cell death mechanisms. Tumour Biol 2010;31:363.

33. Mothersill C, Seymour CB. Radiation-induced bystander effects - implications for cancer. Nat Rev Cancer 2004;4:158-64.

34. Marín A, Martin M, Linan O, et al. Bystander effects and radiotherapy. Rep. Pract. Oncol. Radiother 2014;20:12-21.

35. Zeng J, Harris TJ, Lim M, et al. Immune modulation and stereotactic radiation: improving local and abscopal responses. Biomed Res Int 2013;2013:658126.

36. Kalbasi A, Komar C, Tooker GM, et al. Tumor-derived CCL2 mediates resistance to radiotherapy in pancreatic ductal adenocarcinoma. Clin Cancer Res 2017;23:137-48.

37. Seifert L, Werba G, Tiwari S, et al. Radiation therapy induces macrophages to suppress T-cell responses against pancreatic tumors in mice. Gastroenterology 2016;150:1659-72.e5.

38. Deng L, Liang H, Burnette B, et al. Irradiation and antiPD-L1 treatment synergistically promote antitumor immunity in mice. J Clin Invest 2014;124:687-95.

39. Formenti SC, Demaria S. Systemic effects of local radiotherapy. Lancet Oncol 2009;10:718-26.

40. Schuler G, Steinman RM. Dendritic cells as adjuvants for immune-mediated resistance to tumors. J Exp Med 1997;186:1183-7.

41. Albert ML, Sauter B, Bhardwaj N. Dendritic cells acquire antigen from apoptotic cells and induce class I-restricted 
CTLs. Nature 1998;392:86-9.

42. Galluzzi L, Maiuri MC, Vitale I, et al. Cell death modalities: classification and pathophysiological implications. Cell Death Differ 2007;14:1237-43.

43. Obeid M, Tesniere A, Ghiringhelli F, et al. Calreticulin exposure dictates the immunogenicity of cancer cell death. Nat Med 2007;13:54-61.

44. Demaria S, Formenti SC. Role of T lymphocytes in tumor response to radiotherapy. Front Oncol 2012;2:95.

45. Apetoh L, Ghiringhelli F, Tesniere A, et al. Toll-like receptor 4-dependent contribution of the immune system to anticancer chemotherapy and radiotherapy. Nat Med 2007;13:1050-9.

46. Lugade AA, Sorensen EW, Gerber SA, et al. Radiationinduced IFN- $\gamma$ production within the tumor microenvironment influences antitumor immunity. J Immunol 2008;180:3132-9.

47. Matsumura S, Wang B, Kawashima N, et al. Radiationinduced CXCL16 release by breast cancer cells attracts effector T cells. J Immunol 2008;181:3099-107.

48. Chakraborty M, Abrams SI, Coleman CN, et al. External beam radiation of tumors alters phenotype of tumor cells to render them susceptible to vaccine-mediated T-cell killing. Cancer research 2004;64:4328-37.

49. Demaria $\mathrm{S}, \mathrm{Ng} \mathrm{B}$, Devitt $\mathrm{ML}$, et al. Ionizing radiation inhibition of distant untreated tumors (abscopal effect) is immune mediated. Int J Radiat Oncol Biol Phys 2004;58:862-70.

50. Demaria S, Santori FR, Ng B, et al. Select forms of tumor cell apoptosis induce dendritic cell maturation. J Leukoc Biol 2005 77:361-8.

51. Tang C, Liao Z, Gomez D, et al. Lymphopenia Association With Gross Tumor Volume and Lung V5 and Its Effects on Non-Small Cell Lung Cancer Patient Outcomes. Int J Radiat Oncol Biol Phys 2014;89:1084-91.

52. Zeng J, See AP, Phallen J, et al. Anti-PD-1 blockade and stereotactic radiation produce long-term survival in mice with intracranial gliomas. Int J Radiat Oncol Biol Phys 2013;86:343-9.

53. Sharon E, Polley MY, Bernstein M, et al. Immunotherapy and Radiation Therapy: Considerations for Successfully Combining Radiation into the Paradigm of Immuno-Oncology Drug Development. Radiat Res 2014;182:252-7.

54. Cheever MA, Schlom J, Weiner L, et al. Translational Research Working Group Developmental Pathway for Immune Response Modifiers. Clin Cancer Res 2008;14:5692-9.
55. Cameron RB, Spiess PJ, Rosenberg SA. Synergistic antitumor activity of tumor-infiltrating lymphocytes, interleukin 2, and local tumor irradiation. Studies on the mechanism of action. J Exp Med 1990;171:249-63.

56. Seetharam S, Staba MJ, Schumm LP, et al. Enhanced eradication of local and distant tumors by genetically produced interleukin-12 and radiation. Int J Oncol 1999;15:769-73.

57. Chiang CS, Hong JH, Wu YC, et al. Combining radiation therapy with interleukin-3 gene immunotherapy. Cancer Gene Therapy 2000;7:1172-8.

58. Lumniczky K, Desaknai S, Mangel L, et al. Local tumor irradiation augments the antitumor effect of cytokineproducing autologous cancer cell vaccines in a murine glioma model. Cancer Gene Ther 2002;9:44-52.

59. Chakravarty PK, Alfieri A, Thomas EK, et al. Flt3-Ligand administration after radiation therapy prolongs survival in a murine model of metastatic lung cancer. Cancer Res 1999;59:6028-32.

60. Demaria S, Bhardwaj N, McBride WH, et al. Combining radiotherapy and immunotherapy: a revived partnership. Int J Radiat Oncol Biol Phys 2005;63:655-66.

61. Nikitina EY, Gabrilovich DI. Combination of gammairradiation and dendritic cell administration induces a potent antitumor response in tumor-bearing mice:approach to treatment of advanced stage cancer. Int J Cancer 2001;94:825-33.

62. Teitz-Tennenbaum S, Li Q, Rynkiewicz S, et al. Radiotherapy potentiates the therapeutic efficacy of intratumoral dendritic cell administration. Cancer Res 2003;63:8466-75.

63. Demaria S, Kawashima N, Yang AM, et al. Immunemediated inhibition of metastases following treatment with local radiation and CTLA-4 blockade in a mouse model of breast cancer. Clin Cancer Res 2005;11:728-34.

64. van den Heuvel MM, Verheiji M, Boshuizen R, et al. NHS-IL2 combined with radiotherapy: preclinical rationale and phase $\mathrm{Ib}$ trial results in metastatic non-small cell lung cancer following first-line chemotherapy. J Transl Med 2015;13:32.

65. Linder S, Shoshan MC. Is translational research compatible with preclinical publication strategies? Radiat Oncol 2006;1:4.

66. Golden EB, Chachoua A, Fenton-Kerimian MB, et al. Abscopal Responses in Metastatic Non-Small Cell Lung Cancer (NSCLC) Patients Treated on a Phase 2 Study of Combined Radiation Therapy and Ipilimumab: Evidence for the In Situ Vaccination Hypothesis of Radiation. Int J 
Radiat Oncol Biol Phys 2015;93:S66-7.

67. Pujol JL, Vansteenkiste JF, De Pas TM, et al. Safety and immunogenicity of MAGE-A3 cancer immunotherapeutic with or without adjuvant chemotherapy in patients with resected stage IB to III MAGE-A3-positive non-small-cell lung cancer. J Thorac Oncol 2015;10:1458-67.

68. Vansteenkiste JF, Cho BC, Vanakesa T, et al. Efficacy of the MAGE-A3 cancer immunotherapeutic as adjuvant therapy in patients with resected MAGE-A3-positive nonsmall-cell lung cancer (MAGRIT): a randomised, doubleblind, placebo-controlled, phase 3 trial. Lancet Oncol 2016;17:822-35.

69. Sangha R, Butts C. L-BLP25: A peptide vaccine strategy in non small cell lung cancer. Clin Cancer Res 2007;13:s4652-4.

70. Butts C, Murray N, Maksymiuk A, et al. Randomized phase IIB trial of BLP25 liposome vaccine in stage IIIB and IV non-small-cell lung cancer. J Clin Oncol 2005;23:6674-81.

Cite this article as: Bhalla N, Brooker R, Brada M. Combining immunotherapy and radiotherapy in lung cancer. J Thorac Dis 2018;10(Suppl 13):S1447-S1460. doi: 10.21037/jtd.2018.05.107
71. Butts C, Maksymiuk A, Goss G, et al. Updated survival analysis in patients with stage IIIB or IV non-small-cell lung cancer receiving BLP25 liposome vaccine (L-BLP25): phase IIB randomized, multicenter, open-label trial. J Cancer Res Clin Oncol 2011;137:1337-42.

72. Butts C, Socinski MA, Mitchell P, et al. Tecemotide (L-BLP25) versus placebo after chemoradiotherapy for stage III non-small-cell lung cancer (START): a randomized, double-blind, phase 3 trial. Lancet Oncol 2014;15:59-68.

73. Ohyanagi F, Horai T, Sekine I, et al. Safety of BLP25 liposome vaccine (L-BLP25) in Japanese patients with unresectable stage III NSCLC after primary chemoradiotherapy: Preliminary results from a Phase I/II study. Jpn J Clin Oncol 2011;41:718-22.

74. Antonia SJ, Villegas A, Daniel D, et al. Durvalumab after chemoradiotherapy in stage III non-small-cell lung cancer. N Engl J Med 2017;377:1919-29. 